\title{
Research Paper \\ The Role of Ankle and Knee Joints Proprioceptive Acuity in Improving the Elderly Balance After 8-Week Aquatic Exercise
}

\author{
${ }^{*}$ Ramin Beyranvand $^{1} \oplus$, Mansour Sahebozamani ${ }^{1} \oplus$, Abdolhamid Daneshjoo $^{1} \odot$ \\ 1. Department of Sport Injury \& Corrective Exercises, Faculty of Physical Education, Shahid Bahonar University of Kerman, Kerman, Iran
}

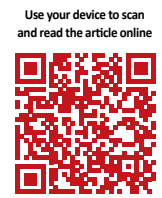

Citation Beyranvand R, Sahebozamani M, Daneshjoo A. [The Role of Ankle and Knee Joints Proprioceptive Acuity in Improving the Elderly Balance After 8-Week Aquatic Exercise (Persian)]. Iranian Journal of Ageing. 2018; 13(3):372-383. https://doi.org/10.32598/SIJA.13.3.372

\section{https://doi.org/10.32598/SIJA.13.3.372}

Keywords:

Proprioceptive acu-

ity, Balance, Aquatic exercise, Elderly

\section{ABSTRACT}

Objectives Proprioception improvement in lower limb joints plays an important role in reducing falling risk in the elderly. Since proprioception improvement in the lower limb joints have the most relevance to better balance in the elderly, this study aimed to investigate the role of ankle and knee joint proprioception on balance improvement in the elderly after an aquatic exercise program.

Methods \& Materials total of 30 elderly male (60-70 years old) were randomly selected and classified into intervention and control groups. Postural sway parameters and proprioception acuity were evaluated using the Biodex balance system and photography method, respectively. Then the intervention group participated in an aquatic exercise program for 8 weeks while the control group did not receive any intervention. All subjects were reassessed upon completion of the intervention program and the Pearson correlation test was used for data analysis $(\mathrm{P} \leq 0.05)$.

Results The results of this study showed a significant relationship between the changes of the ankle joint proprioception and the postural control ability after conducting the 8 weeks of aquatic exercise program $(P<0.05)$; while the above relationship was not significant in the case of the knee joint $(P>0.05)$.

Conclusion It seems that the improvement in ankle joint proprioception plays a more important role in improving the postural control ability of the elderly compared with the other lower limb joints. Accordingly, it is recommended that the special role of the ankle joint should be more considered in the exercise planning for balance improvement in the elderly.

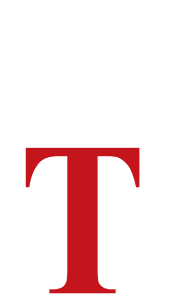

\section{Extended Abstract}

\section{Objectives}

he increasing number of elderly people and the prevalence of physical disabilities in them have drawn the attention of many health and social care systems [1]. In this regard, it is important to study the reduced ability for postural control which is one of the most important and most common problems of the elderly [2,3]. In general, balance is defined as the ability of the body to maintain the center of gravity within the limits of stability [4].

Studies have reported that participation in aquatic exercises to increase the proprioceptive acuity at lower limb joints can play an important role in improving

\footnotetext{
* Corresponding Author:

Ramin Beyranvand, PhD

Address: Department of Sport Injury \& Corrective Exercises, Faculty of Physical Education, Shahid Bahonar University of Kerman, Kerman, Iran Tel: +98 (935) 1098716

E-mail: ramin_beyranvand@yahoo.com
} 
balance in the elderly [5]. However, it has not yet been determined in which lower limb joints the proprioceptive acuity play the most role. In this regard, this study examined and compared the role of the proprioceptive acuity at ankle and knee in improving the elderly balance after aquatic exercise training.

\section{Methods and Materials}

This study is a clinical trial(code:IRCT2016031227022N1). Study population consisted of 80 elderly people living in Kerman City, Iran. Of them, 30 volunteers were randomly selected and divided into experimental $(n=15)$ and control $(n=15)$ group. The inclusion criteria were being male, 60-70 years old and able to walk independently. Subjects who had previous damage or surgery, especially in the lower limbs as well as those with a history of chronic diseases, use of psychotropic medications or participating in a particular sport on a regular basis were excluded from the study.

In order to measure the balance and stability of the subjects, Biodex balance system (ICC $\geq 0.79$ ) was used. Measurement of proprioceptive acuity at ankle and knee joints was performed by photography method (ICC $\geq 0.97$ ). The experimental group intervened by a standard aquatic exercise training protocol for 8 weeks, 3 sessions a week. Each training session consisted of 5 minutes of warm-up, 35 minutes of workout and 5 minutes of cooling down. The control group did not participate in any particular physical activity, and only did their daily routine activities. After 8-week aquatic exercise training, all subjects were reassessed and different stages of measurement were repeated for them. Finally, the collected data were analyzed using Shapiro-Wilk test, Levene's test, Independent t test, combined ANOVA and Pearson correlation test $(\alpha=0.05)$ in SPSS V. 21.

\section{Result}

To determine the homogeneity of experimental and control groups in this study, the Independent $t$ test was used. Results showed no significant difference between groups in terms of age, height, weight and body mass index, and both groups were homogeneous in these respects $(\mathrm{P} \geq 0.05)$. Independent $t$ test was also used to compare the postural control ability as well as proprioceptive acuity between the two groups $(\mathrm{P} \geq 0.05)$. The results showed no significant difference between them and the groups were homogeneous $(\mathrm{P} \geq 0.05)$. In order to evaluate the effect of 8 -week aquatic exercise training on the elderly balance, combined ANOVA was applied.

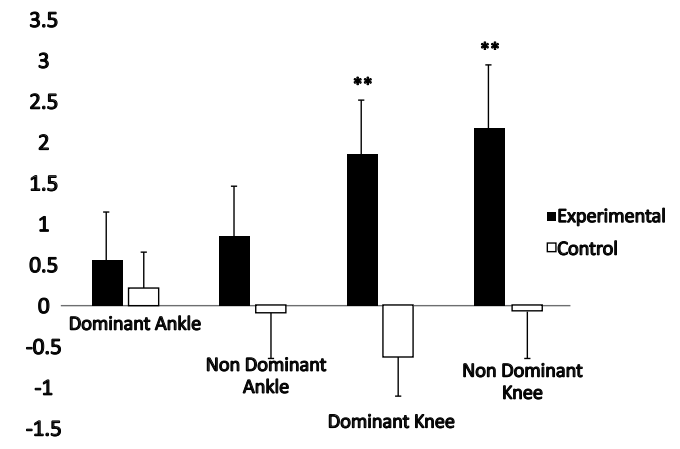

ARGEIN G G

Figure 1. Proprioceptive acuity changes before and after intervention

** $\mathrm{P}<0.05$

Its results indicated that a significant interaction between time (pretest-posttest) and group (control-experimental) in relation to the balance indices $(\mathrm{P}=0.015)$. It was also found that the main effect of time and group on the balance indices of the subjects was significant ( $\mathrm{P}=0.001$ and $\mathrm{P}=0.007$, respectively). Results of proprioceptive acuity changes before and after intervention are shown in Figure 1.

The Pearson correlation test was used to examine the correlation between proprioceptive acuity and postural control ability in the elderly. In this regard, results showed a significant correlation between the changes in ankle joint proprioceptive acuity and the ability for postural control after intervention $(\mathrm{P}=0.031$, and 0.045 for dominant and non-dominant ankles, respectively), while no significant correlation was observed between the changes in knee joint proprioceptive acuity and the postural control ability $(\mathrm{P}=0.136$, and 0.760 for dominant and non-dominant knees, respectively).

\section{Conclusion}

Considering the relationship between ankle joint proprioceptive acuity and the ability for postural control in the elderly men after 8-week aquatic exercise training, it seems that increased accuracy in the proprioceptive acuity of ankle joint is more effective in reducing the oscillations of the elderly's center of gravity compared to the knee joint proprioceptive acuity. As a result, it is recommended that, in training programs for improving the postural control ability of the elderly, the special role of the ankle joint in improving this ability should be taken to into consideration. 


\section{Ethical Considerations}

Compliance with ethical guidelines

The present research was confirmed by the ethics committee of Kerman University of Medical Sciences with the code of IR.KMU.REC.1394.598.

\section{Funding}

This research was extracted from the $\mathrm{PhD}$ thesis of the first author in the Department of Sport Injury \& Corrective Exercises, Faculty of Physical Education, Shahid Bahonar University of Kerman.

\section{Conflict of interest}

The authors declared no conflict of interest.

\section{Acknowledgements}

The authors would like to thank all those who helped us in conducting this research. 


\title{
نقش حس عمقى مج يا و زانو در بهبود تعادل سالمندان يس از يك دوره تمرينات آبى
}

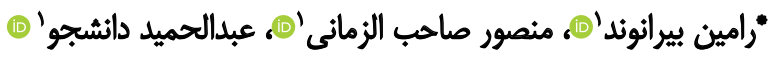

1- كروه آسيبشناسى ورزشى و حركات اصلاحى، دانشكده تربيت بلنى و علوم ورزشى، دانشكاه شهيد باهنر، كرمان، ايران.

\begin{abstract}
حكSد

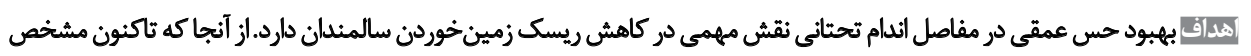

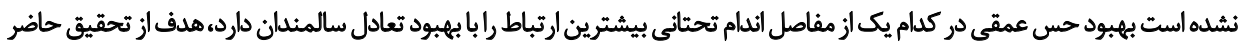

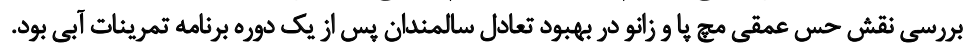

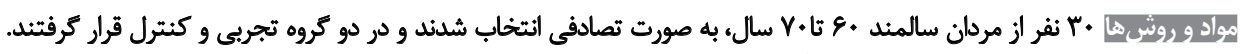

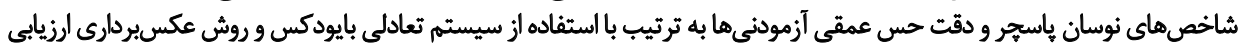

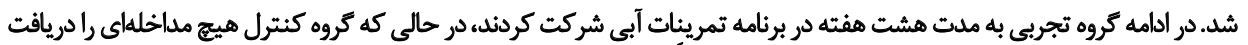

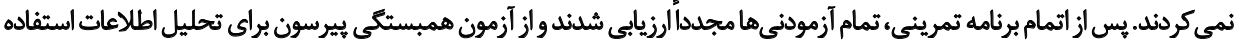

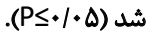

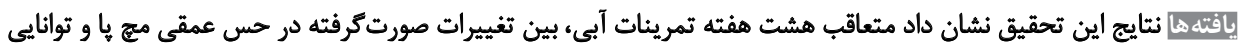

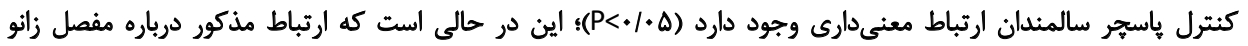

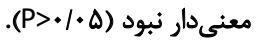

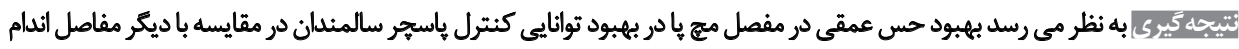

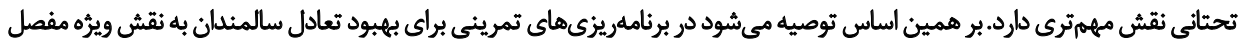

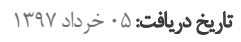

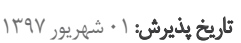

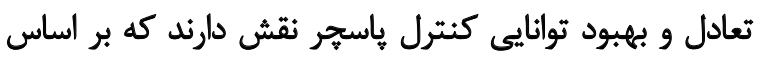

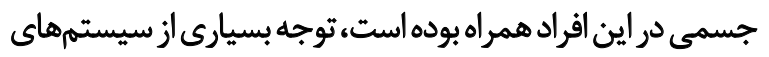

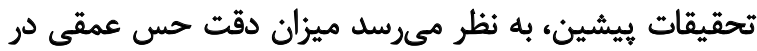

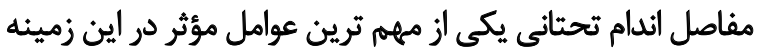

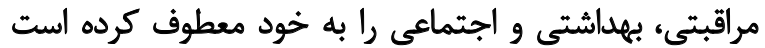

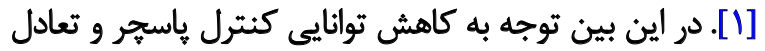

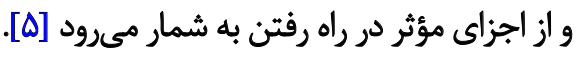

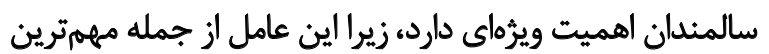

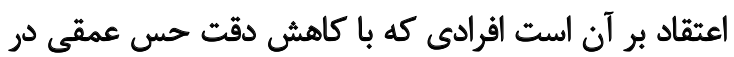

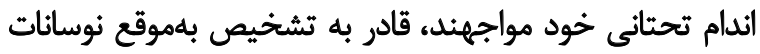

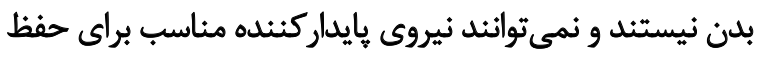

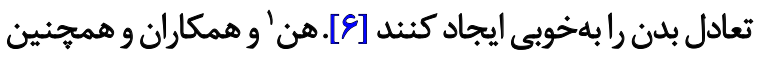

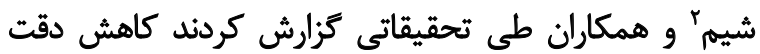

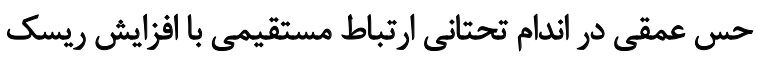

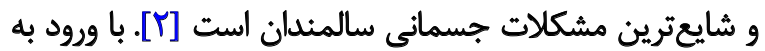

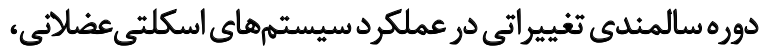

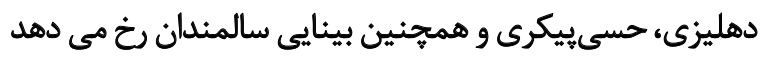

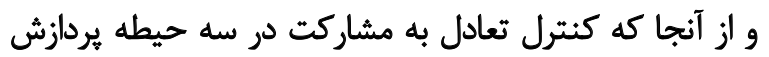

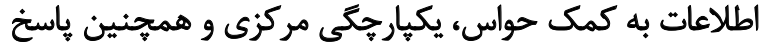

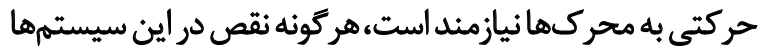

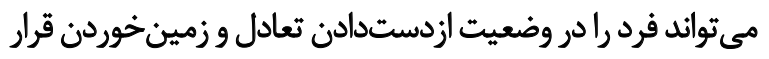

[T]

2. Shim

تعادل و كنترل هاسجحر به عنوان توانايى نكيهدارى مركز ثقل 
كذاشته شدند [IT. IT] غربالكرى افراد با استفاده از يرسشنامه تاريخجه يز يزشكى كه

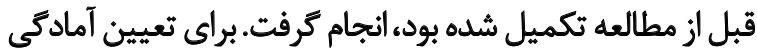

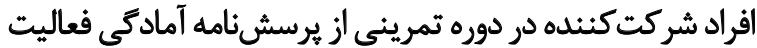

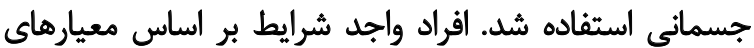

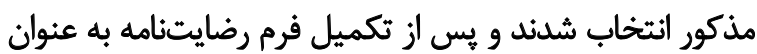
أزمودنى هاى تحقيق بررسى شدند.

اندازهيرى نوسانات قامتى آزمودنىها در تحقيق حاضر باديا

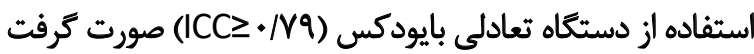

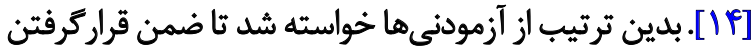

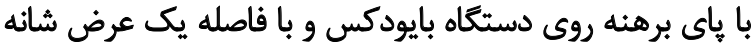

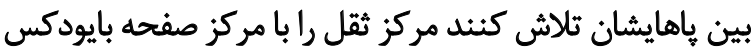

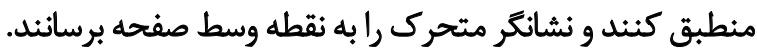

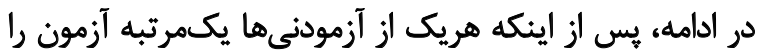

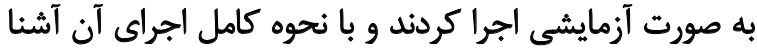

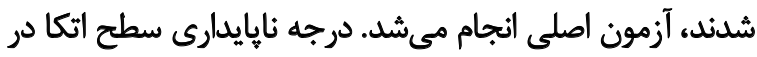

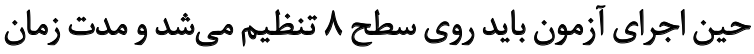

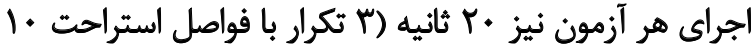

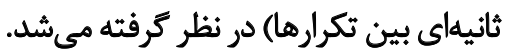

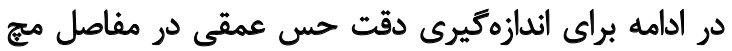

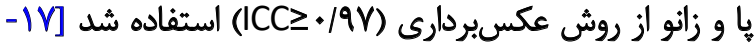

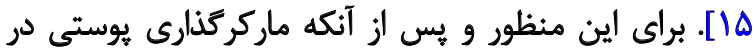

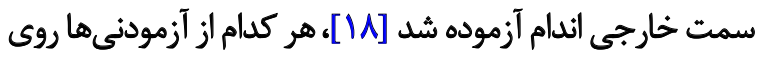

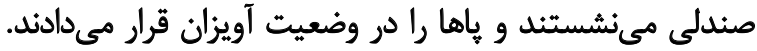

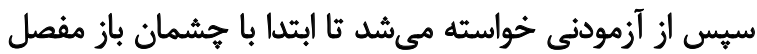

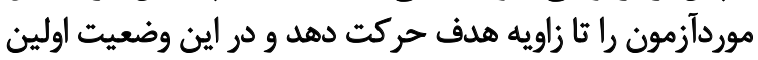

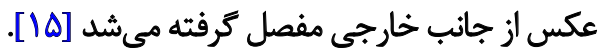

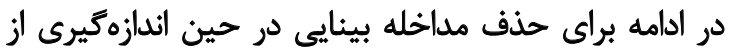

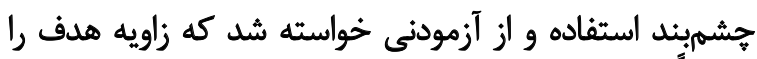

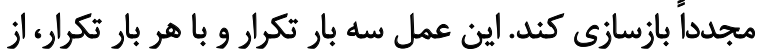

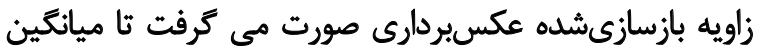

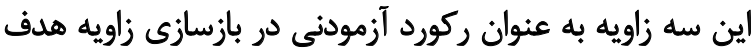

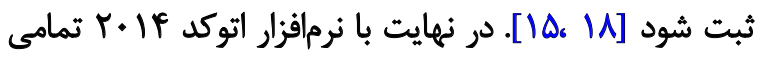

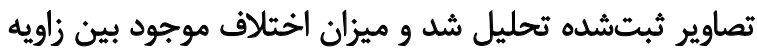

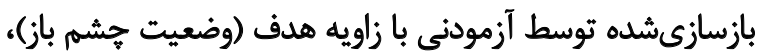

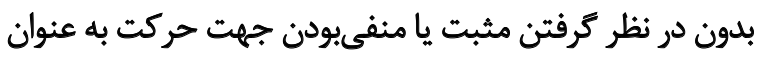

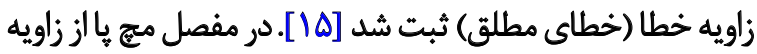

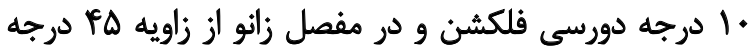

فلكشن به عنوان زاويه هدف استفاده شد [19].

با اتمام بيشآزمون و بعد از فراهم كردن مقدمات كار، كروه

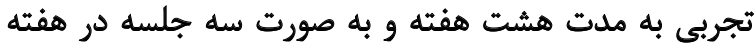

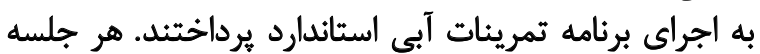

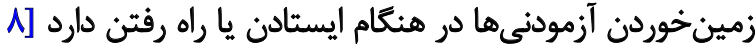

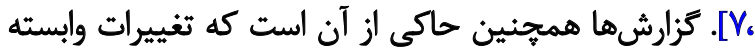

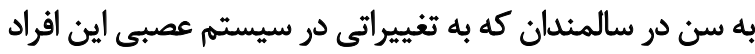

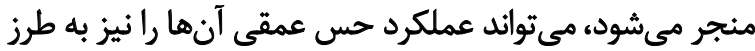

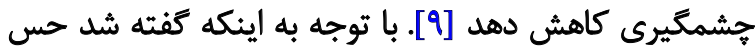

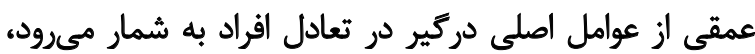

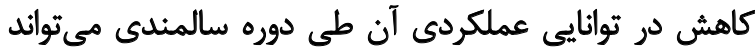

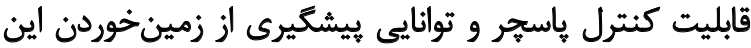

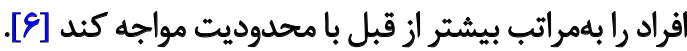

با توجه به مشكلات برشمرده، ثاكنون تحقيقات بسيارى در

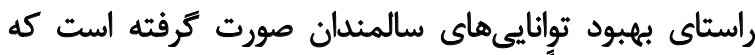

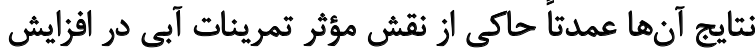

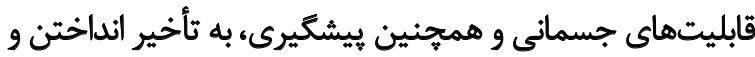

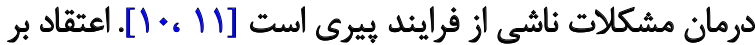

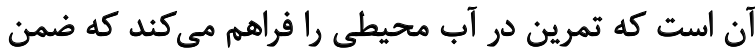

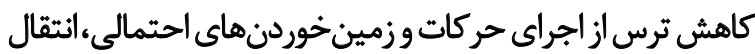

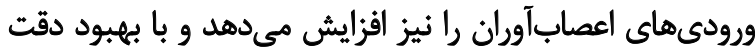

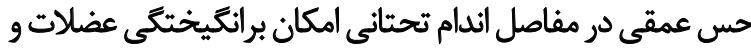

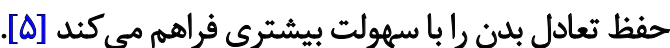
با وجود اينكه نقش تمرينات آبى در بهبود حس عمقى اندام

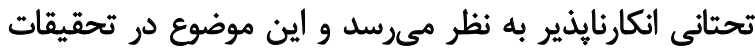

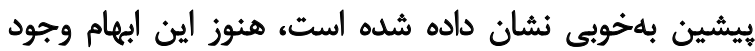

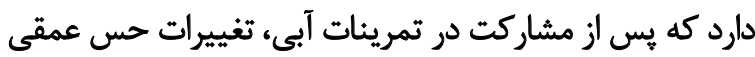

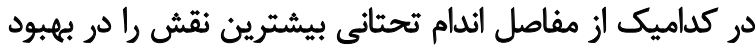

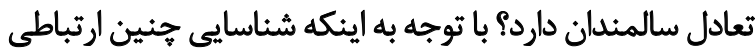

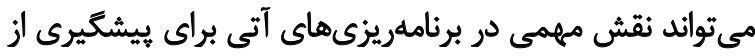

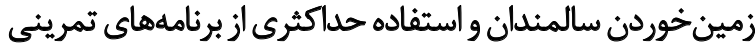

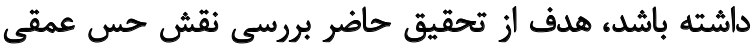

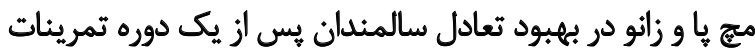

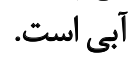

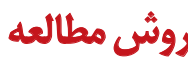

تحقيق حاضر از نوع كارآزمايي بالينى (با كدا

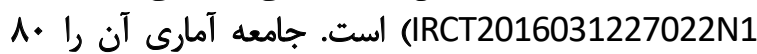

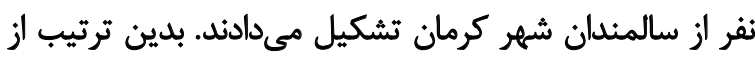

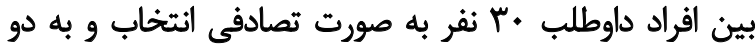

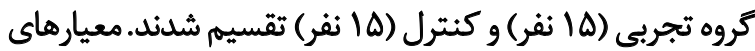

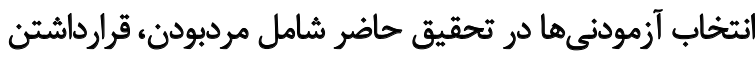

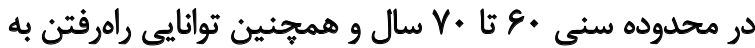

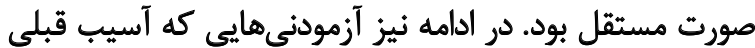

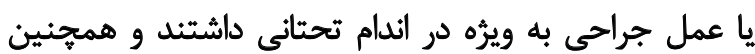

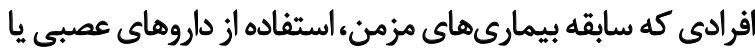
مشاركت در ورزش خاص به طور منظهم را داشتند، از تحقيق كنار 
اين آزمون نيز در جدول شماره الرائه شده است و نشان ميدهد

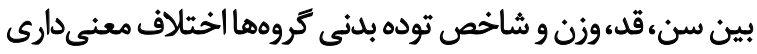

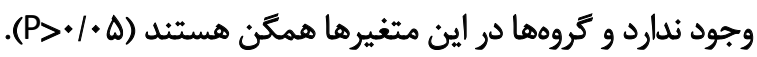

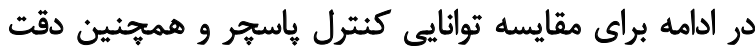

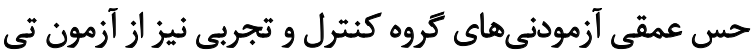

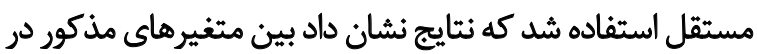

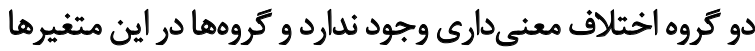

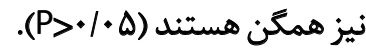
براى بررسى تأثير هشت هفته تمرينات آبى بر نوسانات قامتى آني

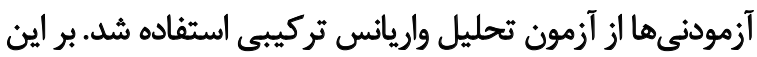

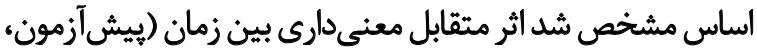

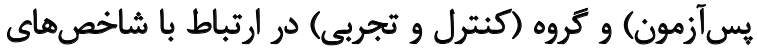

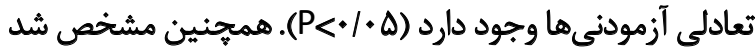

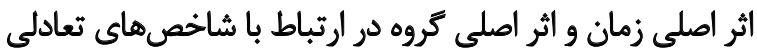

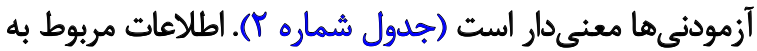

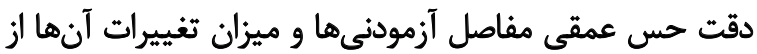

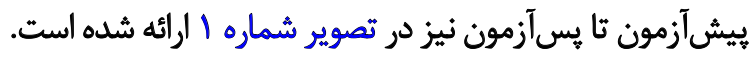
براى بررسى ارتباط بين ميزان تغييرات دقت حس عمقى و

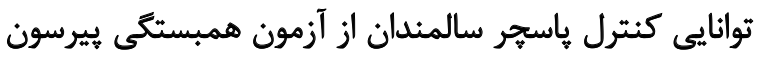

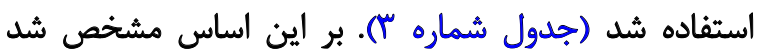

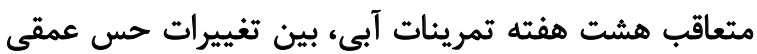

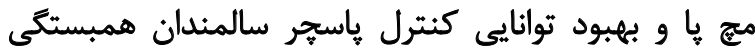

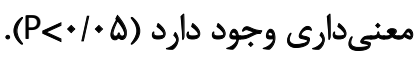

بحث

نتايج بهدستآمده از تحقيق حاضر نشان داد اجراى هشت

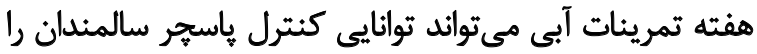

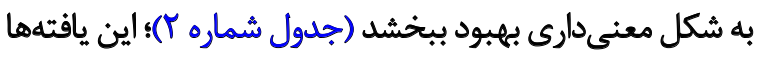

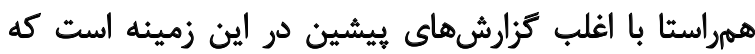

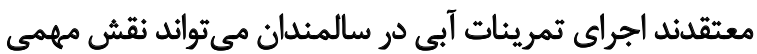

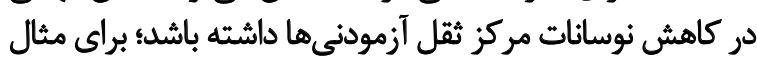

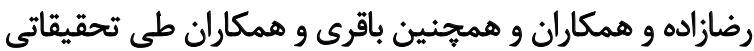

تمرينى شامل بينج دقيقه كرمكردن، ها دقيقه تمرين و وينج

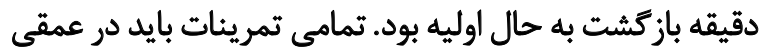

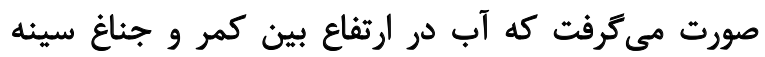

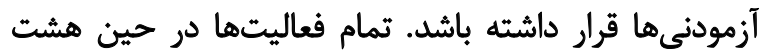

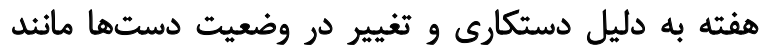

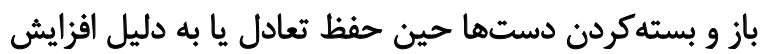

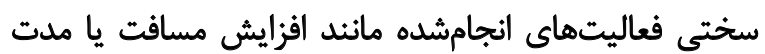

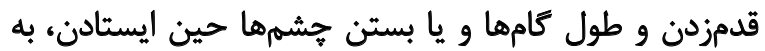

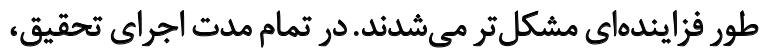

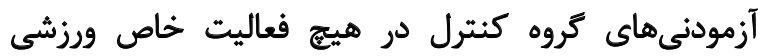

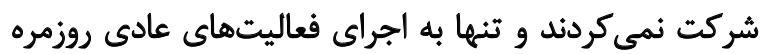

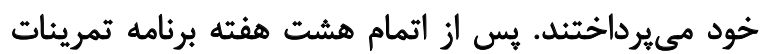

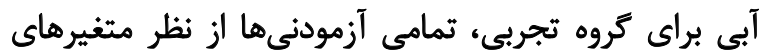

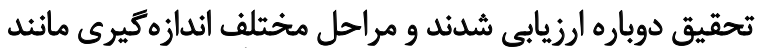

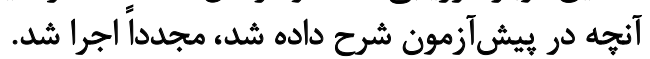

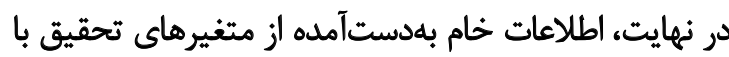

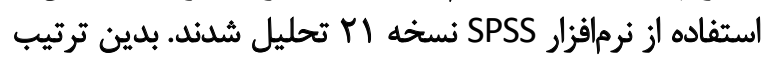

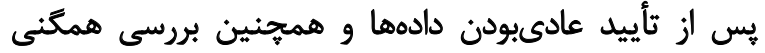

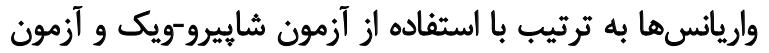

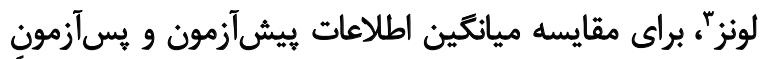

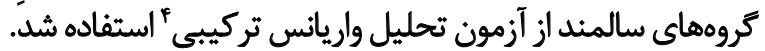

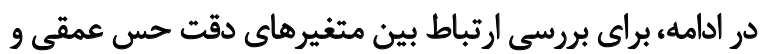

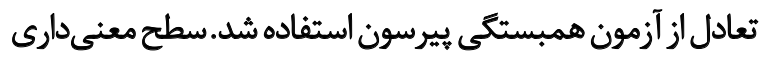

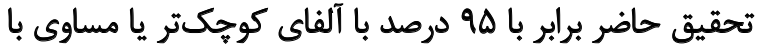

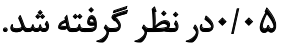

ياقتهلها

اطلاعات جمعيت شناختى آزمودنى ها به تفكيك كروه مربوطه

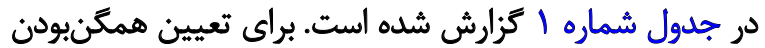
كروهها در اين متغيرها از آزمون تى مستقل استفاده شد كه نت نتايج

3. Levene's

4. Mixed ANOVA

جدول ا. نتايج آزمون تى مستقل براى بررسى وجود اختلاف در مشخصات آنترويومتريكى كروه كنترل و تجربى

\begin{tabular}{|c|c|c|c|c|c|}
\hline \multirow{2}{*}{$\mathbf{P}$} & \multirow{2}{*}{ درجه آزادى } & \multirow{2}{*}{ t آماره } & كروه تجربى & كروه كثترل & \multirow{2}{*}{ مثغير } \\
\hline & & & \multicolumn{2}{|c|}{ Mean \pm SD } & \\
\hline .118 & ra & lefer & $\varepsilon \Delta / F= \pm r / \Delta P$ & $q \pi / \varepsilon \cdot \pm r / r q$ & سن (عال) \\
\hline$\cdot|A| \mid$ & rA & $. / M F \mid$ & $|9 q / \pi r \pm \Delta / 8|$ & $\mid Q N A Y \pm Y / Q E$ & قد (cm) \\
\hline.$/ 948$ & M & $-.1 .+8$ & $V \cdot / A \cdot \pm F / / \% q$ & $V \cdot / q u \pm q / 14$ & وزن \\
\hline . /9Tr & rA & -.1 .99 & $r f / r w \pm r / F q$ & $M T / A T \pm Y / \Delta \Delta$ & شاخص توده بدنى (kg/m²) \\
\hline
\end{tabular}




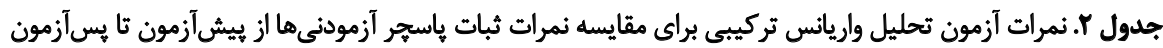

\begin{tabular}{|c|c|c|c|c|c|c|}
\hline & $\mathbf{P}$ & & يسآزمون & ييش آزمون & \multirow{2}{*}{ كروه } & \multirow{2}{*}{ متغير } \\
\hline اثر تعاملى & كروه & زمان & & & & \\
\hline $.1 \cdot 10^{\circ *}$ & $+1 * \gamma^{* *}$ & $.1 . .1^{* *}$ & 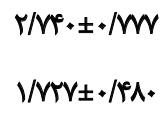 & $\begin{array}{l}r / A V^{\prime} \pm \cdot / 9 r . \\
r / R \Delta Y^{\prime} \pm \cdot / V \cdot r\end{array}$ & تجترل & ثبات كلى \\
\hline
\end{tabular}

بـ

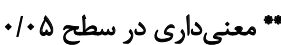

جدول "ا. همبسئكى بين دقت حس عمقى و ميزان تغييرات ثبات ياسجر آزمودنىها

\begin{tabular}{|c|c|c|c|}
\hline $\mathbf{P}$ & $r^{r}$ & ضريب r r & متغير \\
\hline - $\mid \cdot M^{* *}$ &.$/ A Q$ &.$/ 29 \Delta$ & هيج ياي برتر \\
\hline$\cdot 1 \cdot P \theta^{* *}$ & ./Prel & . req & هج ياى غيربرتر \\
\hline / Mre &.$/ . W A$ &.$/ r v a$ & زانوى باى برثر \\
\hline.$/ N 8$ & $\cdot 1 \cdot . \pi$ & $.1 . \Delta A$ & زأوى ياى غيربرتر \\
\hline
\end{tabular}

L

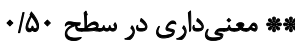

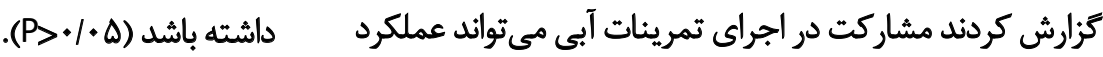

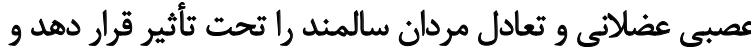
تحليل نتايج همجنين حاكى از آن بود كه بين ميزان تغييرات

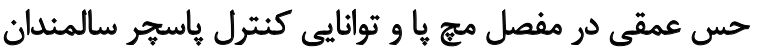

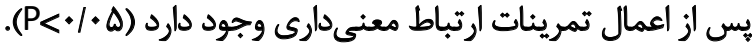

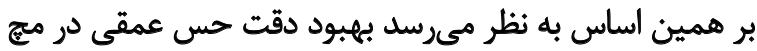

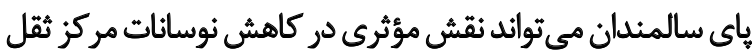

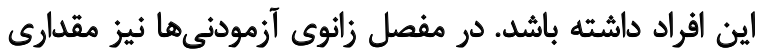

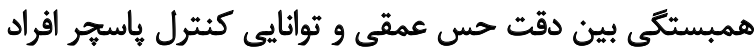

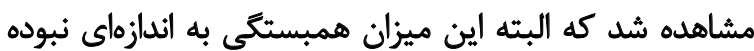

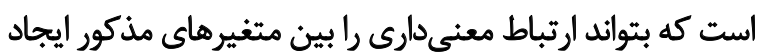

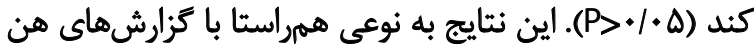

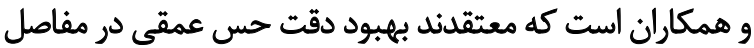

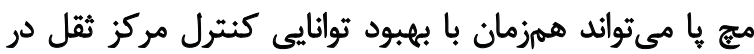

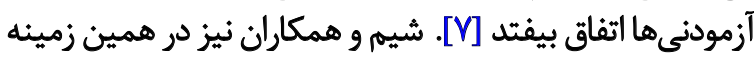

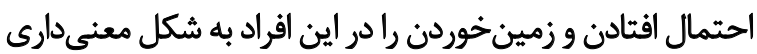

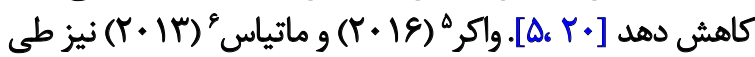

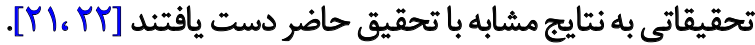
تحقيق حاضر همجنين نشان داد يس از مشاركت سالمندان

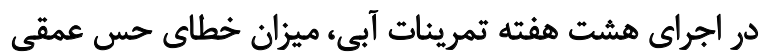

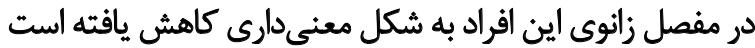

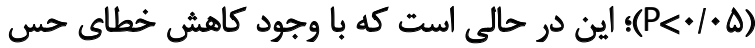

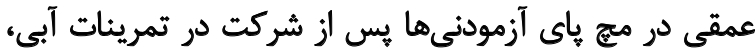

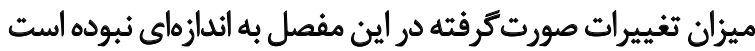

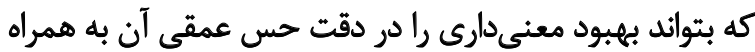

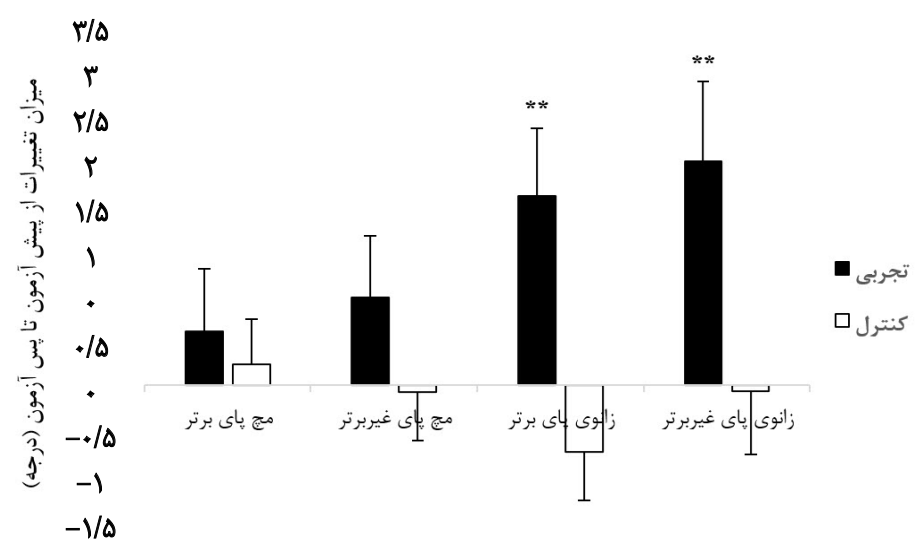


در هر دو سطح هوشيارانه و خودكار شده و زمينه را براي بهبود إنماني

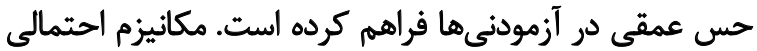

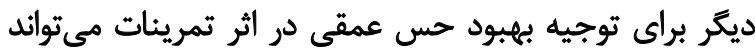

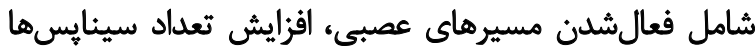

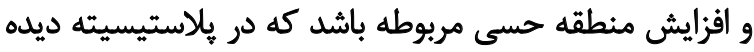

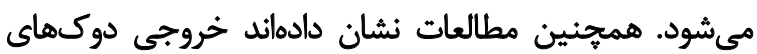

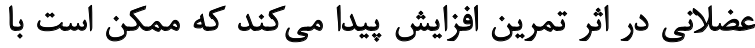

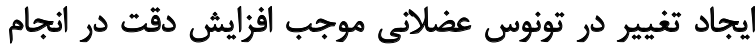

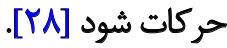

در ارتباط با تغييرات حس عمقى ميج يا در تحقيق حاضر به

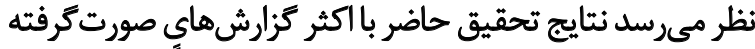

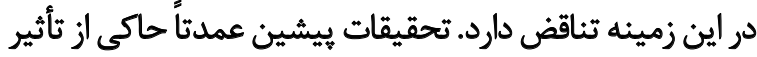

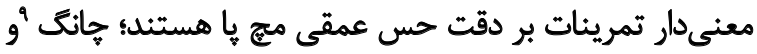

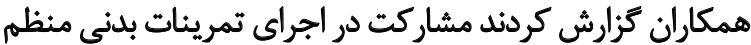

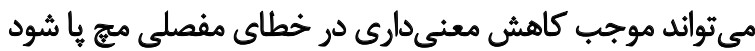

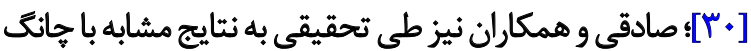

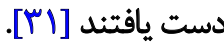

اغلب كزارشهاى موجود در اين زمينه به صورت تمرينات

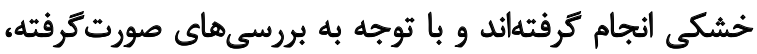

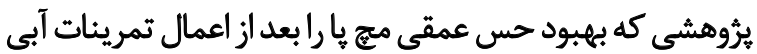

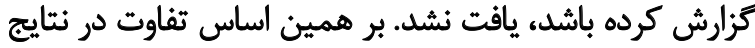

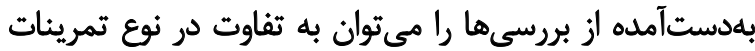

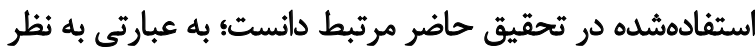

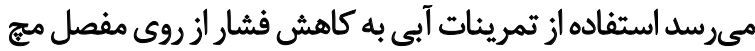

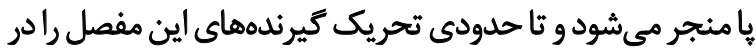

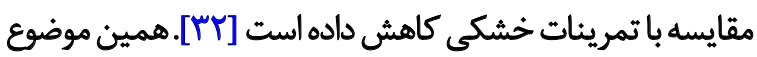
به معنى دار نبودن تغييرات در حس عمقى مج يا يامنجر شده است. درباره دلايل احتمالى ديكر كه ممكن است در تغييرات

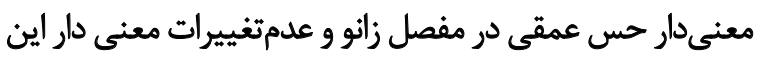

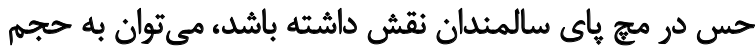

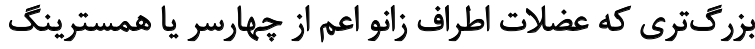

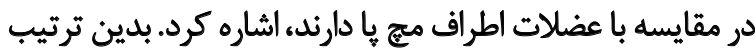

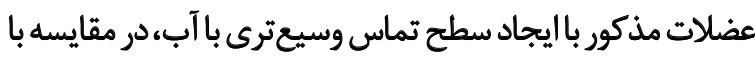

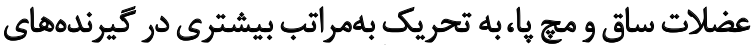

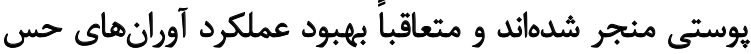

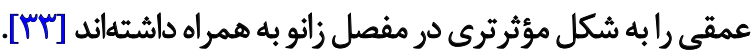

\section{نتيجه تيرى نهايیى}

با توجه به نتايج بهدست آمده از تحقيق حاضر، به نظر ميرسد

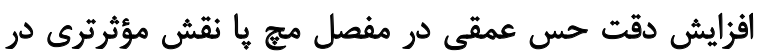

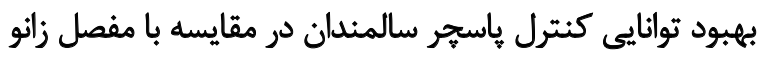

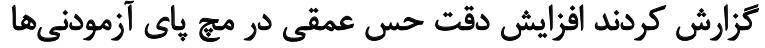

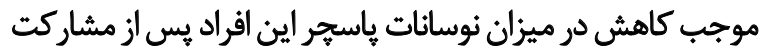

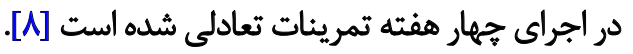

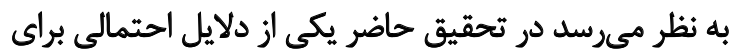

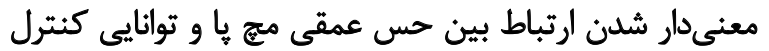

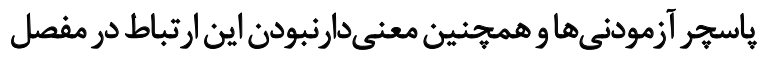

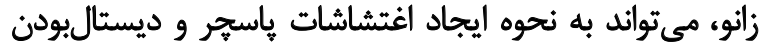

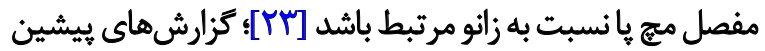

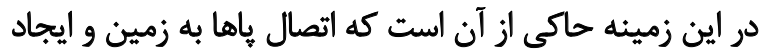

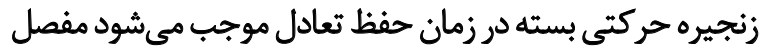

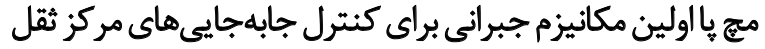

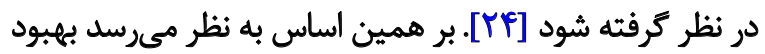

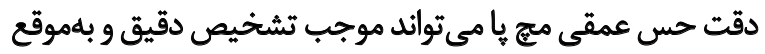

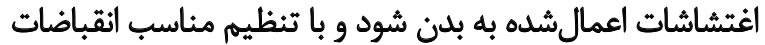

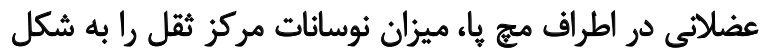

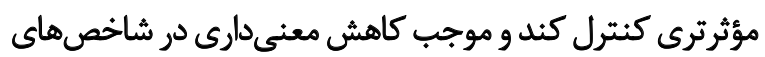

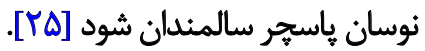
در خصوص تغييرات حس عمقى مفصل زانو در تحقيق حاضر،

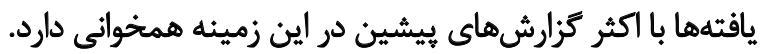

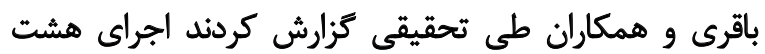

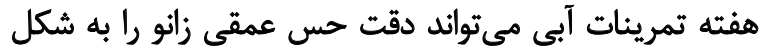

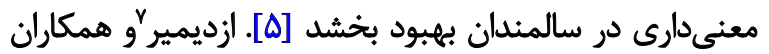

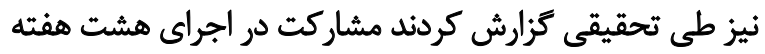

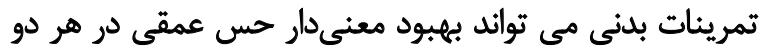

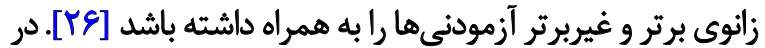

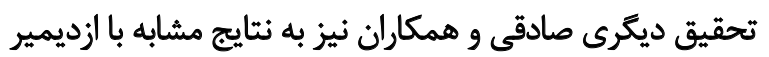

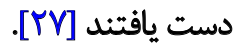

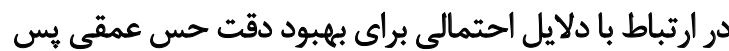

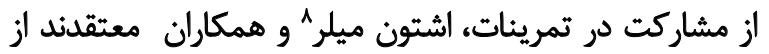

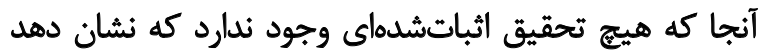

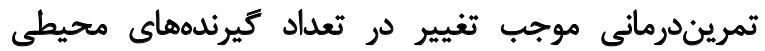

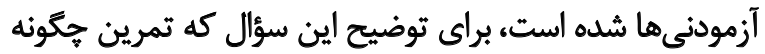

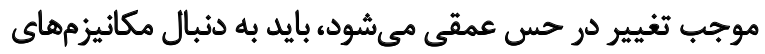

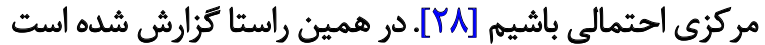

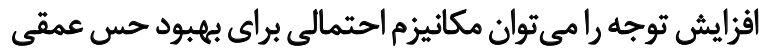

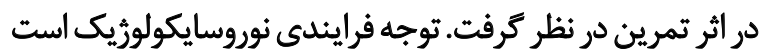

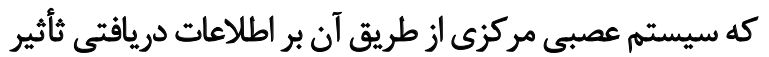
مى تَذارد [rq].

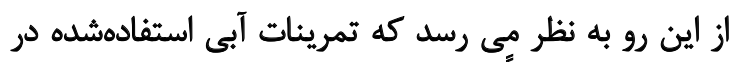
تحقيق حاضر احتمالأ موجب افزايش توجه به به علائم حس عمقى ابنى 
دارد. توصيه ميشود در برنامهريزىهاى تمرينى براى بهبود

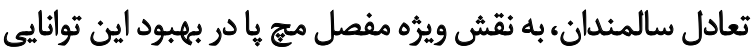

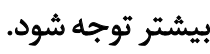

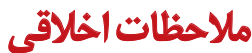

$$
\text { يبروى أز اصول اخلاق ثئوهش }
$$

تحقيق حاضر تأيديد كميته اخلاق دانشكاه علوم يزشكى

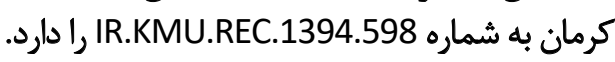

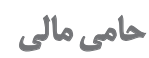

اين مقاله بركرفته از رساله دكترى رامين بيرانوند در رشته آسيبشناسى ورزشى و حركات اصنات اصلاحى دانشكاه شهيد باهنر

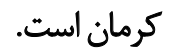

$$
\text { تمعارض مثافع }
$$

بنا بر اظهارنظر نويسندكان اين مقاله تعارض منافع ندارد.

$$
\text { تشكر وقدردانى }
$$

بدين وسيله از تمامى عزيزانى كه در اجراى اين تحقيق مساعدت و همكارى داشتند، تشكر و سياسكزارى مي آشود. 


\section{References}

[1] Abdoli B, Shams A, Shamsipour Dehkordi P. [The effect of practice type on static and dynamic balance in elderly 60-75 year old women with no history of falling (Persian)]. Daneshvar Medicine. 2012; 19(97):43-50.

[2] Almeida S, Soldera C, Carli G, Gomes I, Resende T. Analysis of extrinsic and intrinsic factors that predispose elderly individuals to fall. Revista da Associação Médica Brasileira. 2012; 58(4):427-33. [DOI:10.1016/S0104-4230(12)70224-5]

[3] Shupert C, Horak F. Balance and falls in the older adult. Portland: Vestibular Disorders Association; 2016.

[4] Hof AL, Curtze C. A stricter condition for standing balance after unexpected perturbations. Journal of Biomechanics. 2016; 49(4):580-5. [DOI:10.1016/j.jbiomech.2016.01.021] [PMID]

[5] Bagheri H. [The effect of 8-weeks aquatic exercise on balance strategy and knee joint position sense of 60-70 year old people (Persian)] [MSc. thesis]. Kerman: Shahid Bahonar University of Kerman; 2014.

[6] Ribeiro F, Oliveira J. Aging effects on joint proprioception: The role of physical activity in proprioception preservation. Neurobiology of aging. 2007; 4(2):71-6. [DOI:10.1007/s11556-007-0026-x]

[7] Han J, Anson J, Waddington G, Adams R, Liu Y. The role of ankle proprioception for balance control in relation to sports performance and injury. BioMed Research International. 2015; 2015: 842804. [DOI:10.1155/2015/651048] [PMID] [PMCID]

[8] Shim AL, Steffen K, Hauer P, Cross P, Van Ryssegem G. The effects of balance training on stability and proprioception scores of the ankle in college students. International Journal of Kinesiology \& Sports Science. 2015; 3(4):16-21.

[9] Ambrose AF, Paul G, Hausdorff JM. Risk factors for falls among older adults: A review of the literature. Maturitas. 2013; 75(1):5161. [DOI:10.1016/j.maturitas.2013.02.009] [PMID]

[10] Seyedjafari E, Sahebozamani M, Beyranvand R, Ebrahimipoor E, Razavi M. The effect of 8 weeks deep-aquatic exercises on static balance and lower body strength among elderly men. International Journal of Applied Exercise Physiology. 2017; 6(1):86-98.

[11] Waller B, Ogonowska-Słodownik A, Vitor M, Rodionova $\mathrm{K}$, Lambeck J, Heinonen A, et al. The effect of aquatic exercise on physical functioning in the older adult: A systematic review with meta-analysis. Age and Ageing. 2016; 45(5):593-601. [DOI:10.1093/ageing/afw102] [PMID]

[12] Nobahar Ahari M, Nejati V, Hosseini A. [Comparing ageralated changes of balance performance In youth and older adults (Persian)]. Iranian Journal of Ageing. 2010; 5(1):35-41.

[13] Mahdavi-Ortakvand S, Farzaneh-Hesari A, Zarei M, Khoshdel $M$. [The comparative effects of a gluteus medius strength training program and balance training program on postural control in adult and elder women (Persian)]. Journal of Sabzevar University of Medical Sciences. 2013; 20(3):302-9.

[14] Pereira HM, de Campos TF, Santos MB, Cardoso JR, de Camargo Garcia M, Cohen M. Influence of knee position on the postural stability index registered by the Biodex Stability System. Gait Posture. 2008; 28(4):668-72. [DOI:10.1016/j.gaitpost.2008.05.003] [PMID]
[15] Malekzadeh H, Seidi F, Rajabi R. [Effect of circadian rhythms on knee joint position sense in men college soccer players (Persian)]. Journal of Research in Rehabilitation Sciences. 2015; 11(2):179-90.

[16] Pour Kazemi F, Naseri N, Bagheri H, Fakhari Z. [Reliability of a system consists of skin markers, digital photography, and AutoCAD software for measuring the knee angles (Persian)]. Journal of Modern Rehabilitation. 2009; 2(3):19-24.

[17] Nasseri N, Hadian MR, Bagheri H, Olyaei STG. Reliability and accuracy of joint position sense measurement in the laboratory and clinic; utilising a new system. Acta Medica Iranica. 2007; 45(5):395-404

[18] Stillman B. An investigation of the clinical assessment of joint position sense [PhD thesis]. Melbourne: The University of Melbourne; 2000

[19] Hertel J. Functional anatomy, pathomechanics, and pathophysiology of lateral ankle instability. Journal of Athletic Training. 2002; 37(4):364. [PMID] [PMCID]

[20] Rezazadeh N, Baluchi R. [The effect of 12 weeks exercise in the water and land on the static and dynamic balance and the risk of falling the old people (Persian)]. Iranian Journal of Ageing. 2016; 10(4):140-50.

[21] Matias P, Costa M, Marinho D, Garrido N, Silva A, Barbosa T. Effects of a 12-weeks aquatic training program in body posture and balance. British Journal of Sports Medicine. 2013; 47(10):e3.51e3. [DOI:10.1136/bjsports-2013-092558.55]

[22] Walker C, Oblock A, Dunn M, Vroom K, Hiatt J. The effects of an aquatic exercise program on dynamic and static balance of sedentary. Elders. 2016; 34

[23] Maki BE. Postural strategies. In: Binder MD, Hirokawa N, Windhorst U, editors. Encyclopedia of Neuroscience. Berlin: Springer; 2009. [DOI:10.1007/978-3-540-29678-2_4714]

[24] Koenig J, Puckree T. Injury prevalence, stability and balance among female adolescent soccer players: sport injury. African Journal for Physical Health Education, Recreation and Dance. 2015; 21(1.1):81-91.

[25] Geigle P, Cheek W, Gould M, Hunt H, Shafiq B. Aquatic physical therapy for balance: The interaction of somatosensory and hydrodynamic principles. Journal of Aquatic Physical Therapy. 1997; 5:4-10.

[26] Özdemir N, Subasi S, Gelecek N, Sari S. The Effects of Pilates Exercise Training on Knee Proprioception-A Randomized Controlled Trial. DEÜ Tıp Fakültesi Dergisi. 2009; 23(2):71-9.

[27] Sadeghi H, Hakim MN, Hamid TA, Amri SB, Razeghi M, Farazdaghi $\mathrm{M}$, et al. The effect of exergaming on knee proprioception in older men: A randomized controlled trial. Archives of Gerontology and Geriatrics. 2017; 69:144-50. [DOI:10.1016/j.archger.2016.11.009] [PMID]

[28] Ashton-Miller JA, Wojtys EM, Huston LJ, Fry-Welch D. Can proprioception really be improved by exercises. Knee Surgery, Sports Traumatology, Arthroscopy. 2001; 9(3):128-36. [DOI:10.1007/s001670100208]

[29] Ahmad abadi F, Avandi S. [The effect of four weeks dynamic warm-up on static and dynamic balance and proprioceptive receptors in skilled female Gymnast (Persian)]. Journal of Physiology of Sport and Physical Activity. 2016; 17:1291-300. 
[30] Chang S, Zhou J, Hong Y, Sun W, Cong Y, Qin M, et al. Effects of 24-week Tai Chi exercise on the knee and ankle proprioception of older women. Research in Sports Medicine. 2016; 24(1):84-93. [DOI:10.1080/15438627.2015.1126281] [PMID]

[31] Sadeghi M, Rahnama N, Sadeghi H, Faramarzi M. [Effect of 8 weeks neuromuscular exercise on ankle joint proprioception on male soccer players (Persian)]. Research in Sport Rehabilitation. 2016; 3(6):49-58.

[32] Hall J, Swinkels A, Briddon J, McCabe CS. Does aquatic exercise relieve pain in adults with neurologic or musculoskeletal disease? A systematic review and meta-analysis of randomized controlled trials. Archives of Physical Medicine and Rehabilitation. 2008; 89(5):873-83. [DOI:10.1016/j.apmr.2007.09.054] [PMID]

[33] Douris P, Southard V, Varga C, Schauss W, Gennaro C, Reiss A. The effect of land and aquatic exercise on balance scores in older adults. Journal of Geriatric Physical Therapy. 2003; 26(1):36. [DOI:10.1519/00139143-200304000-00001] 
